\title{
Sinopsis de la producción olivícola peruana: 2005-2011
}

\author{
Synopsis of the peruvian olive production: 2005-2011 \\ Germán F. Sepúlveda-Chavera ${ }^{1 *}$, Ricardo Salvatierra-Martínez ${ }^{1}$, Manuel Rodríguez-Molina ${ }^{1}$
}

\section{RESUMEN}

En Perú, desde 2005 la actividad olivícola muestra un sostenido desarrollo, sustentado por mejoras agronómicas e incorporación de capital, posicionándose competitivamente en la región. La explotación eficiente de una serie de ventajas productivas en el sector olivícola ha permitido proyectar su potencial exportador. En este trabajo se presenta una sinopsis de la producción olivícola de Perú y su impacto en la olivicultura de la Región de Arica y Parinacota, Chile.

Palabras clave: Olivicultura, Olea europaea L., Comercialización.

\begin{abstract}
From 2005 peruvian olive activity shows a important development sustained by agronomic improvements and capital incorporation, resulting in a competitively positioning in the region. The efficient exploitation of a series of productive advantages, allows to project his exporting potential. In this work we presented a synopsis of the Peru olive production, and the possible impact of this development in the olive crop of the Arica and Parinacota region-Chile.
\end{abstract}

Key words: Olive growing, Olea europaea L., Marketing.

\section{Introducción}

El olivo es un árbol cultivado desde la antigüedad por la calidad de sus frutos (Vernet, 1990). Su centro de origen se localiza desde el sur del Cáucaso hasta las planicies de Irán, Palestina y la zona costera de Siria poblando la cuenca del Mediterráneo (Civantos, 2000). Desde allí se extendió a América, cultivándose actualmente también en Sudáfrica, China, Japón y Australia (Wiesman, 2009). La expansión global de este cultivo se puede explicar en atención a su rusticidad, plasticidad ambiental (escasos requerimientos de horas de frío y tolerancia a las heladas), tolerancia a déficit hídrico, salinidad y adaptación a diferentes tipos de suelo. De acuerdo con Hidalgo (1993), llegó a América a fines del siglo XVI, estableciéndose en el sector "La Chimba”, en Arica para ocupar el valle de Azapa, Chile (Wormald, 1972). En Perú se estableció durante la Colonia en los valles de Yauca, Atiquipa e Ilo (Hidalgo, 1993), permaneciendo en un estado de poco desarrollo hasta principios del siglo XXI. Desde 2000 en adelante, la actividad olivícola del Perú muestra un sostenido desarrollo, sustentado por mejoras agronómicas, incorporación de capital y mejoramiento productivo, lo que se tradujo en un importante posicionamiento competitivo en la región. La producción olivícola se concentra principalmente en el Sur siendo el Departamento de Tacna la zona de mayor crecimiento. Desde el año 2000 a 2005 éste creció de una superficie cultivada de 3.200 ha a una superficie de 4.890 ha, siendo el primer productor peruano de olivos (Casapía et al., 2005).

El objetivo de este trabajo es presentar una sinopsis de la producción olivícola de Perú y visualizar el impacto de este desarrollo en la olivicultura del valle de Azapa, Chile.

\section{Metodología}

Para estudiar la producción olivícola de Perú se trabajó con un estudio descriptivo, recabando antecedentes agronómicos y comerciales de las exportaciones de aceituna peruana en los principales mercados mundiales. Se determinaron las características competitivas del rubro olivícola, observando las relaciones que se producen entre ellas de acuerdo con una investigación no experimental de tipo

\footnotetext{
1 Universidad de Tarapacá. Arica, Chile. Av. General Velásquez 1775, Casilla 6-D, Arica.

* Autor para correspondencia: gsepulve@uta.cl
} 
documental y tendencia longitudinal (Hernández et al., 1998). Se analizaron las tendencias a través del tiempo, en cuanto a países compradores y volúmenes comercializados. Finalmente se infirieron tendencias en base al comportamiento observado, sus determinantes y consecuencias en el sistema olivícola de la Región de Arica y Parinacota, Chile. Se trabajó con fuentes de información secundaria obtenidas de diferentes bases de datos. Se recopiló información estableciendo series entre los años 2005-2011. Los valores registrados en las exportaciones se obtuvieron a partir de bases de datos del Ministerio de Agricultura de Perú (MINAG), y de Chile (MINIAGRI), Consultores privados (MAXIMIXE, TradeMap, Global Trade Atlas), Registros y estadísticas de ingresos de productos desde Perú en el Servicio Agrícola y Ganadero (SAG) entre 2007 y 2011.

\section{Resultados y Discusión}

\section{Producción olivícola}

Según cifras del Ministerio de Agricultura de Perú se cultivan 10.000 ha de olivos principalmente en la zona sur (Tacna, Arequipa, Ica y Moquegua). Tacna, capital del departamento homónimo, representa más del $60 \%$ de la producción de aceitunas y es la zona que obtiene mayor rendimiento, con $32 \mathrm{mil}$ toneladas métricas (MINAG-Perú, 2011a), debido a una eficiente conducción técnica y aplicación de buenas prácticas agrícolas (MAXIMIXE, 2011). Desde 2007 la producción de Tacna corresponde al $68 \%$ y Arequipa al $27 \%$ de la producción del país (PROSAAMER, 2008). Otras zonas productoras son Moquegua con 3\%, Ica y Lima con $2 \%$ (MINAG-Perú, 2011b).

\section{Caracterización de productores}

Los productores olivícolas peruanos se pueden clasificar en dos tipos: el agricultor tradicional y el agricultor moderno (Cuadro 1).

\section{Producción de aceituna de mesa}

Con apoyo de la cooperación técnica española y una fuerte orientación estatal, el crecimiento de la producción de aceitunas en Perú alcanzó un significativo nivel cualitativo y cuantitativo

\begin{tabular}{cl}
\hline Agricultor tradicional & Comercializa mayoritariamente sin pro- \\
& cesar y se orienta al mercado interno. El \\
producto adolece de selección (tamaño, & color), presenta carozo grande por el \\
& uso de variedades aceiteras, y presenta \\
& manchas en los frutos. La conducción \\
& técnica tradicional incorpora poda, \\
& fertilización, control de malezas, riegos, \\
& etc. Los huertos presentan baja o nula \\
& asistencia técnica, poca capacidad de \\
& inversión, gerencial y poco desarrollo \\
& de habilidades de exportación. Además, \\
& existe poca experiencia en manejo hídrico \\
& y en fertilización de los huertos. Este \\
& sistema productivo se orienta a mantener \\
& rendimientos máximos y costos mínimos. \\
& Produce aceitunas de alta calidad \\
& dirigida principalmente al mercado \\
& externo. Las principales empresas \\
& exportadoras cuentan con integración \\
& tecnológica vertical y horizontal, y \\
& tienen todo el proceso de elaboración de \\
& aceitunas: procesamiento, calibración y \\
& almacenamiento. Posee alta tecnología \\
& en fertilización, manejo de plagas y \\
& malezas. Recibe asistencia técnica en \\
el cultivo y en el proceso postcosecha. & Tiene alta capacidad gerencial. \\
\hline &
\end{tabular}

Fuente: modificado de MAXIMIXE Consult S.A., 2011

(Figura 1) y un fuerte posicionamiento comercial de sus exportaciones, especialmente en Brasil, EE.UU., Japón y Chile. Los acuerdos comerciales internacionales suscritos por Perú con diferentes países, incluyendo Chile, permitieron el acceso de la producción peruana a nuevos mercados y promovieron la reducción de cuotas de mercado a sus competidores, tales como Chile y Argentina. Estas oportunidades se fundamentan principalmente en el bajo costo de la mano de obra, entre otros factores productivos.

\section{Exportación de aceitunas}

En 2011 el valor FOB de las exportaciones de aceitunas en sus diferentes presentaciones sumó US\$ 32 millones, lo que representa un aumento de 122 por ciento en comparación con el mismo periodo del 2010, que fue de US\$ 14 millones. El principal país de destino fue Brasil, que recibió el $60 \%$ de lo exportado. Le siguieron Venezuela con 13\%; Chile con 11,4\% y Estados Unidos con 10,2\% (MINAG-Perú, 2011b). 


\section{Exportación de aceitunas en salmuera de Perú}

Para la presentación Aceitunas en Salmuera, Chile es el principal importador con casi 4 millones de dólares en valor FOB (TradeMap, 2012). Según cifras del Servicio Agrícola y Ganadero (SAG, 2012) desde el año 2007 han ingresado 21 millones de kilogramos de aceitunas de la categoría indicada, solo en 2011, 7 millones de kilogramos, aumentando en 148\% desde el 2007 (Figura 2). Esta cantidad es significativa si se considera que el potencial productivo de la Región de Arica y Parinacota (valle de Azapa) se estima en 10 millones de kilogramos anuales sin considerar las fluctuaciones ocurridas por el fenómeno de "El Niño". El ingreso de aceituna en salmuera a Chile ha tenido un efecto muy negativo en las ventas de aceitunas del valle de Azapa, sobre todo en el pequeño productor dedicado a la venta a granel de aceituna para el mercado nacional.

Otro destino importante para la Aceituna en Salmuera de Perú es Venezuela con 530 mil dólares FOB y 273 mil kilogramos del producto. En tercer lugar se ubica Estados Unidos con 306 mil dólares FOB y 129 mil kilogramos (Tabla 1). En términos de participación de las exportaciones de aceituna de mesa de Perú, Chile representa el principal país, obteniendo una cuota de exportaciones del 54,8\%, y una tasa de crecimiento anual en el periodo 2006-2010 de 15\%, lo siguen Venezuela con una cuota del $26,96 \%$ y una tasa del $8 \%$, Brasil con una cuota de exportación del $13,71 \%$ y una tasa de crecimiento de $-27 \%$. Estados Unidos presenta una cuota de $4,29 \%$ y una tasa de crecimiento de $82 \%$ (TradeMap, 2012).

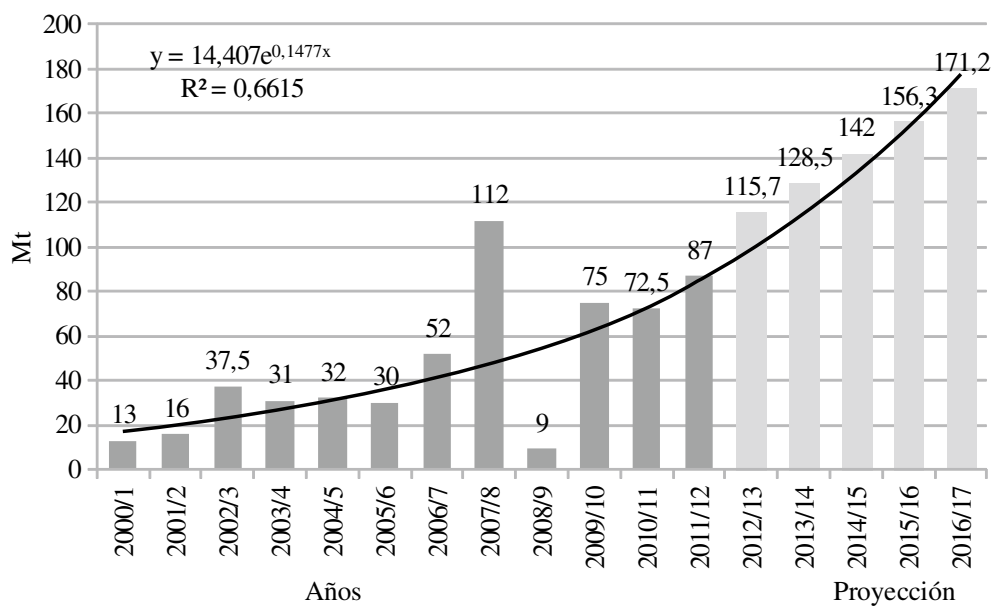

Figura 1. Producción de aceitunas en Perú, período 2000/1-2011/12 y proyección 2013-2017. Adaptado de datos del GTA.

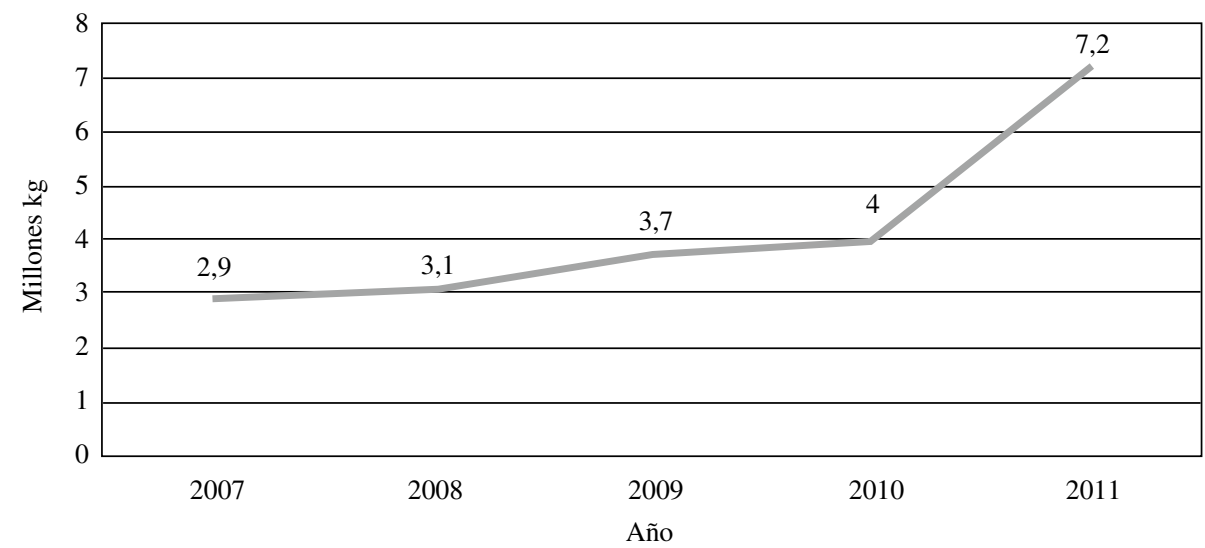

Figura 2. Aceitunas ingresadas a Chile desde Perú por el complejo fronterizo Chacalluta. Fuente: Servicio Agrícola y Ganadero, SAG. 
Tabla 1. Principales países importadores de Aceitunas en Salmuera (cod. 071120), desde Perú, período 2009-2011 (Elaborado en base a Global Trade Atlas, 2012).

\begin{tabular}{|c|c|c|c|c|c|c|}
\hline \multirow[b]{3}{*}{ País Socio } & \multicolumn{6}{|c|}{ AÑO } \\
\hline & \multicolumn{2}{|c|}{2009} & \multicolumn{2}{|c|}{2010} & \multicolumn{2}{|c|}{2011} \\
\hline & USD & Cantidad & USD & Cantidad & USD & Cantidad \\
\hline Chile & 858.884 & 1.422 .510 & 2.402 .117 & 3.540 .852 & 3.905 .735 & 6.221 .018 \\
\hline Venezuela & 780.757 & 446.125 & 919.170 & 501.945 & 530.470 & 273.265 \\
\hline Estados Unidos & 110.406 & 41.708 & 188.450 & 76.047 & 306.783 & 129.657 \\
\hline Brasil & 66.072 & 29.700 & 601.049 & 434.700 & 111.750 & 77.900 \\
\hline Duty Free & 235.971 & 241.598 & 191.512 & 362.100 & 99.000 & 254.794 \\
\hline Colombia & 0 & 0 & 0 & 0 & 57.450 & 32.400 \\
\hline Canadá & 200 & 40 & 195 & 45 & 3.382 & 937 \\
\hline Aruba & 1.192 & 185 & 0 & 0 & 73 & 24 \\
\hline Francia & 26.433 & 14.850 & 0 & 0 & 18 & 3 \\
\hline
\end{tabular}

Tabla 2. Principales países importadores de Aceitunas Preparadas o Conservadas (cod. 200570) de Perú (Elaborado en base a Global Trade Atlas, 2012).

\begin{tabular}{|c|c|c|c|c|c|c|}
\hline \multirow{3}{*}{ País Socio } & \multicolumn{6}{|c|}{ AÑOS } \\
\hline & \multicolumn{2}{|c|}{2009} & \multicolumn{2}{|c|}{2010} & \multicolumn{2}{|c|}{2011} \\
\hline & USD & Cantidad & USD & Cantidad & USD & Cantidad \\
\hline Brasil & 14.052 .058 & 9.915 .904 & 24.144 .139 & 14.122 .418 & 15.463 .862 & 8.800 .309 \\
\hline Estados Unidos & 2.569 .340 & 948.671 & 3.339 .517 & 1.060 .632 & 2.679 .184 & 735.134 \\
\hline Venezuela & 928.791 & 420.902 & 817.085 & 355.786 & 1.426 .731 & 590.688 \\
\hline Chile & 1.690 .450 & 2.234 .496 & 2.061 .013 & 2.607 .746 & 1.142 .243 & 1.367 .789 \\
\hline Francia & 415.066 & 205.295 & 407.685 & 179.006 & 648.221 & 276.145 \\
\hline Ecuador & 91.851 & 38.047 & 113.861 & 34.969 & 317.384 & 59.642 \\
\hline Canadá & 293.816 & 117.025 & 325.989 & 130.159 & 219.734 & 87.271 \\
\hline Kuwait & 108.459 & 50.104 & 128.608 & 49.952 & 154.070 & 62.860 \\
\hline Rusia & 0 & 0 & 27.277 & 13.999 & 152.593 & 26.997 \\
\hline Colombia & 157.472 & 96.012 & 108.001 & 64.038 & 127.707 & 78.948 \\
\hline
\end{tabular}

Tabla 3. Principales exportadores de Aceituna de Mesa de Perú

(Fuente: Departamento de Exportaciones Agrícolas de Perú ADEX, 2012).

\begin{tabular}{llccc}
\hline $\mathrm{N}^{\mathrm{o}}$ & \multicolumn{1}{c}{ Ranking de empresas exportadoras } & US\$ FOB millones 2010 & US\$ FOB millones 2009 & $\%$ Var. \\
\hline 01 & Agroindustrias Nobex S.A. & 11,3 & 8,1 & 39.5 \\
02 & Agroind. y Comercializ. Guive E.I.R.L & 2,6 & 1,4 & 85.7 \\
03 & Biondi y CIA de Tacna S.A.C. & 1,0 & 1,2 & -16.7 \\
04 & Exportadora El Sol S.A.C. & 1,5 & 1,2 & 25.0 \\
05 & R Muelle S.A. & 0,8 & 1,1 & -27.3 \\
06 & Corporación Mirki S.A. & 0,0 & 0,9 & -100.0 \\
07 & Exportaciones Mirsa E.I.R.L. & 2,2 & 0,8 & 175.0 \\
08 & Almacenes S.A.C. & 0,1 & 0,8 & -87.5 \\
09 & Sihersa S.A. & 0,8 & 0,7 & 14.3 \\
10 & Descals Industrias Alimentarias S.A.C. & 1,1 & 0,7 & 57.1 \\
11 & Armano Olives S.A.C. & 1,1 & 0,7 & 57.1 \\
12 & Agroindustrias Los Andes S.A.C. & 0,6 & 0,6 & 0.0 \\
13 & Okiva Perú S.A.C. & 0,9 & 0,6 & 50.0 \\
14 & Truffa Corporation S.A.C. & 1,3 & 0,4 & 225.0 \\
15 & Cia. Agroindustrial Punta del Sur S. R. Ltda. & 0,6 & 0,3 & 100.0 \\
16 & Inversiones Yauca E.I.R.L. & 0,6 & 0,3 & 100.0 \\
17 & Frutos y granos del Perú S.A.C. & 0,9 & 0,3 & 200.0 \\
\hline
\end{tabular}




\section{Exportación de Perú de aceitunas preparadas o conservadas}

Las exportaciones de este producto desde el mercado peruano se orientan a los mercados de Brasil y Estados Unidos. El primer lugar lo ocupa el mercado brasileño con 15 millones de dólares en valor FOB y 8 millones de kilogramos al año 2011 (Tabla 2). Lo siguen Estados Unidos y Venezuela. En cuarto lugar se observa a Chile con un millón de dólares en valor FOB y un millón de kilogramos correspondientes al año 2011 (TradeMap, 2012). Brasil tiene la principal cuota de exportaciones de Perú de aceitunas preparadas o conservadas, correspondiendo a $74,54 \%$, con una tasa de crecimiento de importaciones anual en los periodos 2006-2010 del 19\%. En períodos anteriores, este nicho era ocupado por aceitunas producidas en Chile. Lo siguen Estados Unidos con una cuota del $10,3 \%$ y una tasa de crecimiento anual de $4 \%$. Chile representa una cuota de exportaciones del $6,3 \%$ y una tasa de crecimiento anual de $17 \%$ (TradeMap, 2012).

De acuerdo con la información disponible en el Departamento de Exportaciones Agrícolas de Perú (ADEX DataTrade, 2012), 17 empresas realizan envíos al extranjero (Tabla 3). Agroindustrias Nobex S. A. lidera las exportaciones de aceituna de mesa del mercado peruano al exterior, con envíos de 11,3 millones de dólares. Agroindustria y Comercializadora Guive EIRL y Exportaciones Mirsa EIRL tienen exportaciones de 2,6 y 2,2 millones de dólares, respectivamente.

El perfil olivícola de Perú presenta, sin embargo, poca fidelización en los mercados, poca madurez en el proceso exportador y escaso valor agregado al producto (Gobierno Local Inclán, 2012). A esto se debe agregar bajo nivel de desarrollo de la cadena productiva del olivo en diferentes distritos originado en el bajo nivel tecnológico ya mencionado para el agricultor tradicional, desarticulación de los agentes productivos y deficiente comercialización de la producción olivícola. Esto último, sumado al desarrollo de nuevas tecnologías que optimicen los costos productivos de sus competidores (Chile y Argentina) y con ello un aumento de la producción, podría limitar el desarrollo olivícola de Perú (MAXIMIXE, 2011). Sin embargo, Perú está haciendo los esfuerzos necesarios para superar las deficiencias señaladas y posicionándose como un productor relevante en la región.

Desde el punto de vista agrícola la competitividad se ve demostrada por el desarrollo de tecnologías que permitan mayor producción y disminución en los costos de los procesos productivos (Vargas y Tapia, 2008). El Perú presenta ventajas competitivas respecto de zonas productoras como el valle de Azapa dadas por disponibilidad y bajos costos de mano de obra y disponibilidad de agua. Sumado esto a la orientación estatal le permite aprovechar su potencial exportador.

\section{Agradecimientos}

Los autores agradecen al proyecto P058, Código 30110595-0 "Mejoramiento tecnológico del rubro olivícola 1.0: camino a la diferenciación productiva del rubro olivícola", impulsado por el GORE Arica y Parinacota y a la Universidad de Tarapacá por el soporte financiero.

\section{Bibliografía}

ADEX-Perú DataTrade, Exportaciones de aceitunas http://www. adexperu.org.pe/ Consultado: 12/06/12

Calderón L., L.O.

2008 Diagnóstico de la Cadena Productiva del Olivo. 21 pp. http://www.agritacna.gob.pe/tacna/publicaciones2007/ diagnostico-cadena-olivo-hasta-2008.pdf / Consultado: $02 / 12 / 12$.

Casapia, E.; Navarro, L.; Noriega, R.; Saenz, M.

2005 Estrategias para el desarrollo del sector olivícola peruano. Ed. CENTRUM, $1^{\text {ra }}$ Ed. Lima-Perú

Civantos, L.

2000 La olivicultura en el mundo y en España. In: Barranco, Fernández-Escobar y Rallo (Eds.). El cultivo del olivo. Mundi-Prensa. España. 17-33.
Global Trade Atlas (GTA) Import/export Word statistics. http:// www.gtis.com/GTA/ Consultado: 12/06/12.

Gobierno Local Inclán.

2012 Proyecto "Mejoramiento de la Producción Olivícola en el Distrito de Inclán - Provincia de Tacna - Región Tacna". Oficina de Formulación de Proyectos y Estudios. Gobierno Local Inclán. 157 pp.

Hernández, R.; Fernández, C.; Baptista, P. 1998 Metodología de la Investigación, Editorial McGrawHill, México.

Hidalgo, J.

1993 Algunas notas para la historia del olivo en Arica. IDESIA 12: $31-50$. 
International Trade Map. Trade statistics for international business development http://www.trademap.org/ Consultado: 12/06/12. Ministerio de Agricultura Perú (MINAG)

2011 "Ministro de Agricultura, Miguel Caillaux, apoyará a Tacna y Moquegua en ampliación de frontera agrícola" Unidad de Imagen Institucional. http://www.minag.gob. pe/portal/notas-de-prensa/notas-de-prensa-2011/5983ministro-de-agricultura-miguel-caillaux-apoyara-a-tacna-ymoquegua-en-ampliacion-de-frontera-agricola Consultado: $12 / 07 / 12$.

Ministerio de Agricultura Perú (MINAG)

2011 "Exportaciones de aceitunas sumaron US\$ 3,2 millones en enero registrando un aumento de $122 \%$ ". Unidad de Imagen Institucional. http://www.minag.gob. pe/portal/notas-de-prensa/notas-de-prensa-2011/5251 exportaciones-de-aceitunas-sumaron-us-32-millones-enenero-registrando-un-aumento-de-122 Consultado: 12/07/12.

\section{PROSAAMER}

2008 Cadena productiva del olivo en Tacna. Ministerio de Agricultura. 20 pp. Moquegua-Perú.

Servicio Agrícola y Ganadero (SAG)

2012 Documento oficial en respuesta a solicitud N ${ }^{\circ}$ AR006P1500008 [Santiago, 16 de marzo 2012].
Vargas, M.; Tapia, L.

2008 Competitividad de las exportaciones de aceitunas de mesa (Olea europea L.) chilena en el mercado brasileño entre los años 1993-2005. Idesia 26(1):49-57.

Vernet, J.L.

1990 Man and vegetation in the Mediterranean area during the last 2000 years. In: F. di Castro, A. J. Hansen and M. Debusche (Eds.). Kluwer Ac. Press. Dodrecht. Netherlands.

Wiesman, Z.

2009 Chapter 18 - The future of desert olive oil, and concluding remarks. In: Zeev Wiesman. Desert Olive Oil Cultivation, Academic Press. 387-394 pp.

Wormald Cruz, A.

1972 Historias olvidadas del norte grande. Depto. Historia y Geografía. Universidad del Norte. 298 pp.

Maximixe Consult S.A.

(s/f) Perfil del Mercado Competitividad Exportadora de Aceitunas. Base de datos, Ministerio de Comercio Exterior y Turismo (MICETUR) Lima Perú http://www.mincetur. gob.pe/comercio/otros/penx/pdfs/Aceituna.pdf Consultado: $12 / 08 / 12$. 